\title{
SUPPLEMENT TO: RELATIVE ERRORS FOR BOOTSTRAP APPROXIMATIONS OF THE SERIAL CORRELATION COEFFICIENT
}

\author{
By Chris Field ${ }^{*}$ AND John Robinson ${ }^{\dagger}$ \\ Dalhousie University and University of Sydney
}

We give proofs for some results in Field and Robinson (2013).

1. Proofs of the Barndorff-Nielsen approximation. We give proofs for the saddlepoint tail probability approximations for means of independent but not identically distributed random variables when the cumulant generating function, $K_{n}(t)$, exists only in a region $0 \leq t<H<\infty$. This extends the results of $\mathrm{Hu}$, Petrov and Robinson (2007) and uses standard methods which appear not to have been given in this general case.

1.1. Indirect Edgeworth expansions. Let $X_{1}, X_{2}, \cdots$ be independent random variables and define

$$
K_{n}(t)=\frac{1}{n} \log E e^{n t \bar{X}}=\frac{1}{n} \sum_{j=1}^{n} \log E e^{t X_{j}} .
$$

Assume that, for some $H>0$,

$$
K_{n}(t)<\infty \text { for } 0 \leq t<H,
$$

Consider the associated random variables with distribution functions

$$
F_{X_{j t}}(x)=\int_{-\infty}^{x} e^{t y} d F_{X_{j}}(y) / \int_{-\infty}^{\infty} e^{t y} d F_{X_{j}}(y),
$$

and $\bar{X}_{t}=\frac{1}{n} \sum_{j=1}^{n} X_{j t}$. Then

$$
\begin{aligned}
F_{\bar{X}_{t}}(x) & =\int_{-\infty}^{x} e^{n t y} d F_{\bar{X}}(y) / \int_{-\infty}^{\infty} e^{n t y} d F_{\bar{X}}(y) \\
& =e^{-n K_{n}(t)} \int_{-\infty}^{x} e^{n t y} d F_{\bar{X}}(y)
\end{aligned}
$$

* Supported by NSERC Discovery Grant

${ }^{\dagger}$ Supported by ARC DP0773345

AMS 2000 subject classifications: Primary 62G09,62G10,62G20; secondary 62M10

Keywords and phrases: Saddlepoint approximations, autoregression

imsart-aos ver. 2011/12/06 file: ARsprevsup1.tex date: March 11, 2013 
and

$$
F_{\bar{X}}(x)=e^{n K_{n}(t)} \int_{-\infty}^{x} e^{-n t y} d F_{\bar{X}_{t}}(y) .
$$

Let $m_{n t}=\frac{1}{n} \sum_{j=1}^{n} E X_{j t}=K_{n}^{\prime}(t)$ and $\sigma_{n t}^{2}=\frac{1}{n} \sum_{j=1}^{n} \operatorname{var}\left(X_{j t}\right)=K_{n}^{\prime \prime}(t)$. In the sequel take $C$ as a constant and $\theta$ as a quantity bounded by $C$ both of which may differ on each occurrence. Assume

(2) $\sigma_{n}^{2} \rightarrow \sigma^{2}$, as $n \rightarrow \infty$ and $M_{n}=\frac{1}{n} \frac{\sum_{i=1}^{n} E\left(X_{i}-m_{n}\right)^{4}}{\sigma_{n}^{4}}<C<\infty$,

where $\sigma^{2}>0$. Note that, from (1), (2) implies, for $0 \leq t<H$,

(3) $\sigma_{n t}^{2} \rightarrow \sigma_{t}$, as $n \rightarrow \infty$ and $M_{n t}=\frac{1}{n} \sum_{j=1}^{n} \frac{E\left(X_{j t}-m_{n t}\right)^{4}}{\sigma_{n t}^{4}}<C<\infty$,

where $\sigma_{t}^{2}>0$. The characteristic function of $\sqrt{n}\left(\bar{X}_{t}-m_{n t}\right) / \sigma_{n t}$ is

$$
e^{n\left(K_{n}\left(t+1 \xi / \sigma_{n t} \sqrt{n}\right)-n K_{n}(t)-i \xi m_{n t} / \sigma_{n t} \sqrt{n}\right)} .
$$

Assume that for $0<q<1,0 \leq t<H$ and $c \sqrt{n}<\xi<C n$

(4) $\left|e^{n K_{n}\left(t+i \xi / \sigma_{n t} \sqrt{n}\right)-n K_{n}(t)}\right|=\left|\prod_{j=1}^{n} E e^{\left(t+i \xi / \sigma_{n t} \sqrt{n}\right) X_{j}} / \prod_{j=1}^{n} E e^{t X_{j}}\right|<q^{n}$.

Let $G_{\bar{X}_{t}}(x)=\Phi\left(x^{*}\right)+\varphi\left(x^{*}\right) \kappa_{3 n t} H_{2}\left(x^{*}\right) / 3 ! \sqrt{n}$ be the one term Edgeworth expansion of $F_{\bar{X}_{t}}(x)=P\left(\bar{X}_{t}<x\right)$, where $x^{*}=\sqrt{n}\left(x-m_{n t}\right) / \sigma_{n t}$ and $\kappa_{3 n t}$ is the third cumulant of $\sqrt{n}\left(\bar{X}_{t}-m_{n t}\right)$. Write

$$
\bar{G}_{\bar{X}}(x)=e^{n\left(K_{n}(t)-t m_{n t}\right)} \int_{x}^{\infty} e^{-n t\left(y-m_{n t}\right)} d G_{\bar{X}_{t}}(y) .
$$

Choose $t$ so that $m_{n t}=K_{n}^{\prime}(t)=x$, so $x_{t}^{*}=0$, where $x$ is chosen so that $m_{n t}<H$. Changing the variable in the integral in (5), first to $u=\sqrt{n}(y-$ $x) / \sigma_{n t}$ and then to $v=u+\lambda$ for $\lambda=\sqrt{n} t \sigma_{n t}$, leads to

$$
\bar{G}_{\bar{X}}(x)=e^{n\left(K_{n}(t)-t x\right)} \tau(\lambda)\left(1+\rho_{3}(\lambda) \kappa_{3 n t} / 6 \sqrt{n}\right),
$$

where

$$
\tau(\lambda)=\frac{e^{\lambda^{2} / 2}}{\sqrt{2 \pi}} \int_{\lambda}^{\infty} e^{-v^{2} / 2} d v
$$

and

$$
\begin{aligned}
\rho_{3}(\lambda) \tau(\lambda) & =\frac{e^{\lambda^{2} / 2}}{\sqrt{2 \pi}} \int_{\lambda}^{\infty} e^{-v^{2} / 2} H_{3}(v-\lambda) d v \\
& =(-\lambda)^{3} \tau(\lambda)+\left(\lambda^{2}-1\right) / \sqrt{2 \pi}
\end{aligned}
$$


Note that these functions are defined in Robinson et al. (1990), in particular, the last equality is from Appendix B, and they are related to the Esscher functions from Section 2.2 of Jensen (1995).

The following theorem now follows by applying Theorem 1 of Robinson et al. (1990), as in their Remark 2 and Corollary 2.4.

Theorem 1. Assume (1), (3) and (4) and that t, the solution of $K_{n}^{\prime}(t)=$ $x$, satisfies $0 \leq t<H$. Then,for $\lambda=\sqrt{n} t \sigma_{n t}$,

$$
\left.\bar{F}_{\bar{X}}(x)=e^{n\left(K_{n}(t)-t x\right)}\left[\tau(\lambda)\left(1+\rho_{3}(\lambda) \kappa_{3 n t} / 6 \sqrt{n}\right)\right)+\theta M_{n} / n\right] .
$$

1.2. The Barndorff-Nielsen approximation. We show that

$$
I=\int_{x}^{\infty} \frac{e^{-n\left(t(y) y-K_{n}(t(y))\right)}}{\sqrt{2 \pi K_{n}^{\prime \prime}(t(y)) / n}} d y=\bar{\Phi}\left(\sqrt{n} w^{+}\right)\left(1+\theta M_{n} / n\right),
$$

where $w^{+}=w-\log \psi(w) / n w$ for $t(y) y-K_{n}\left(t(y)=w^{2} / 2\right.$, where $t(y)$ is the solution of $y=K_{n}^{\prime}(t)$. Further, we show that the integral in (7) equals the right hand side of $(6)$, where we write $t=t(x)$.

Make the change of variable $v=\left\{2\left(t(y) y-K_{n}(t(y))\right)\right\}^{1 / 2}$ and define $\psi(v)=v / t(y)\left\{K_{n}^{\prime \prime}(t(y))\right\}^{1 / 2}$, then

$$
\begin{aligned}
I & =\int_{w}^{\infty} \frac{e^{-(v-\log \psi(v) / n v)^{2} / 2}}{\sqrt{2 \pi / n}} e^{\log ^{2} \psi(v) / 2 n v^{2}} d v \\
& =\int_{x^{+}}^{\infty} \frac{e^{-u^{2} / 2}}{\sqrt{2 \pi / n}} e^{\log ^{2} \psi(v) / 2 n v^{2}} \frac{d v}{d u} d u,
\end{aligned}
$$

after the transformation $u=v-\log \psi(v) / n v$, where $w=\sqrt{2\left(t x-K_{n}^{\prime \prime}(t)\right)}$ and $w^{+}=w-\log \psi(w) / n w$. Now,

$$
\frac{d u}{d v}=1+\frac{\log \psi(v)}{n v^{2}}-\frac{\psi^{\prime}(v)}{n v \psi(v)}
$$

so, expanding the second and third terms of the integrand in (8) about 0 , in the same manner as in Section 3.3 of Jensen (1995), leads to (7).

Now consider the expansion of the integrand in $I$ about $x$. Let $z=\sqrt{n}(y-$ $x) / \sqrt{K_{n}^{\prime \prime}(t)}$, then

$$
\begin{aligned}
I= & e^{-n\left(t x-K_{n}(t)\right)} \int_{0}^{\infty} \frac{e^{-n\left[(y-x) t-(y-x)^{2} \sigma_{n t}^{2} / 2-(y-x)^{3} K_{n}^{\prime \prime \prime}(t)+(y-x)^{4} \theta M_{n}\right]}}{\sqrt{2 \pi / n} \sigma_{n t}\left(1-(y-x) K_{n}^{\prime \prime \prime}(t)+(y-x)^{2} \theta M_{n}\right)} d y \\
= & e^{-n\left(t x-K_{n}(t)\right)} \int_{0}^{\infty} \frac{e^{\sqrt{n} z t \sigma_{n t}-z^{2} / 2}}{\sqrt{2 \pi}}\left(1+H_{3}(z) \kappa_{3 n t} / 6 \sqrt{n}+\theta M_{n} / n\right) d z . \\
& \text { imsart-aos ver. 2011/12/06 file: ARsprevsup1.tex date: March 11, } 2013
\end{aligned}
$$


This is in the form that led to (5), so $I$ can be written as in (6), thus completing the proof of the following theorem.

Theorem 2. Let $X_{1}, X_{2}, \ldots$ be defined as before and satisfy the conditions of Theorem 1 and let $t$ be the solution of $K_{n}(t)=x$, then

$$
P(\bar{X} \geq x)=\bar{\Phi}\left(\sqrt{n} w^{+}\right)(1+O(1 / n)) .
$$

Finally, let us use the notation of Field and Robinson (2013). Then, conditionally on $\mathbf{C}$, if $E X_{1}^{8}<C, A_{i} X_{2 i}-u\left(X_{2 i}^{2}+B_{i}\right)$ are independent and satisfy the conditions of Theorem 2 , so the equation (11) and the equivalent result for the bootstrap in Field and Robinson (2013) hold.

\section{Proofs of Lemmas of Field and Robinson (2013).}

2.1. Proof of Lemma 1. Consider first

$$
\int h(z) g\left(z \mid X_{2 i-1}, X_{2 i+1}\right) d z \text {. }
$$

For notational convenience consider the case $i=1$ and note that $X_{2}=$ $\rho_{0} X_{1}+\epsilon_{2}$ and $X_{3}=\rho_{0} X_{2}+\epsilon_{3}$, and we can rewrite these equations as

$$
\zeta=\frac{\epsilon_{2}-\rho \epsilon_{3}}{\sqrt{1+\rho_{0}^{2}}}=\sqrt{1+\rho_{0}^{2}}\left(X_{2}-\frac{\rho_{0}\left(X_{1}+X_{3}\right)}{1+\rho_{0}^{2}}\right)
$$

and

$$
\xi=\frac{\rho_{0} \epsilon_{2}+\epsilon_{3}}{\sqrt{1+\rho_{0}^{2}}}=\frac{X_{3}-\rho_{0}^{2} X_{1}}{\sqrt{1+\rho_{0}^{2}}} .
$$

Now $X_{1}$ is independent of $\epsilon_{2}$ and $\epsilon_{3}$, so the conditional distribution of $X_{2}-$ $\rho_{0}\left(X_{1}+X_{3}\right) /\left(1+\rho_{0}^{2}\right)$ given $X_{1}, X_{3}$ is the conditional distribution of $\zeta$ given $\xi$. Thus

$$
\int h(z) g\left(z \mid X_{1}, X_{3}\right) d z=\int h\left(\zeta+\frac{\rho_{0}\left(X_{1}+X_{3}\right)}{\sqrt{1+\rho_{0}^{2}}}\right) f(\zeta \mid \xi) d \zeta
$$

Now consider

$$
\int h(z) g^{\dagger}\left(z \mid X_{2 i-1}^{\dagger}, X_{2 i+1}^{\dagger}\right) d z=\frac{N_{i}^{\dagger}}{\Delta_{i}^{\dagger}}
$$

where, from (16) of Field and Robinson (2013) and the results following that equation,

$$
\Delta_{1}^{\dagger}=\frac{1}{(n-1)^{2}} \sum_{k} \sum_{l} e^{-\left(X_{3}^{\dagger}-\rho_{0}^{2} X_{1}^{\dagger}-\rho_{0} \eta_{k}-\eta_{l}\right)^{2} / 2 \tau^{2}\left(1+\rho_{0}^{2}\right)}
$$

imsart-aos ver. 2011/12/06 file: ARsprevsup1.tex date: March 11, 2013 
and

$$
N_{1}^{\dagger}=\sum_{k} \sum_{l} \int \frac{h(z)}{2 \pi \tau^{2}(n-1)^{2}} e^{-\frac{\left(1+\rho_{0}^{2}\right)\left(z^{\prime}-\left(\eta_{k}-\rho_{0} \eta_{l}\right) /\left(1+\rho_{0}^{2}\right)\right)^{2}}{2 \tau^{2}}-\frac{\left(X_{3}^{\dagger}-\rho_{0}^{2} X_{1}^{\dagger}-\rho_{0} \eta_{k}-\eta_{l}\right)^{2}}{2 \tau^{2}\left(1+\rho_{0}^{2}\right)}} d z \text {. }
$$

Recall that $\eta_{k}=\left(\epsilon_{k}-\bar{\epsilon}\right) / \sigma_{n}$. Note that we can take $\sigma_{n}=1$ by incorporating it with $\tau$, so both $N_{1}^{\dagger}$ and $\Delta_{1}^{\dagger}$ are of the form

$$
\begin{aligned}
\frac{1}{(n-1)^{2}} \sum_{k} \sum_{l} \ell\left(\eta_{k}, \eta_{l}\right)= & \frac{1}{(n-1)(n-2)} \sum_{k \neq l}\left[\ell\left(\epsilon_{k}, \epsilon_{l}\right)\right. \\
& \left.-\bar{\epsilon}\left(\ell_{10}\left(\epsilon_{k}, \epsilon_{l}\right)+\ell_{01}\left(\epsilon_{k}, \epsilon_{l}\right)\right)\right]+O_{P}(1 / m) .
\end{aligned}
$$

The first term here is a $U$-statistic (see for example Chapter 5 of Serfling (1980)) and we can write

$$
\frac{1}{(n-1)(n-2)} \sum_{k} \sum_{l} \ell\left(\epsilon_{k}, \epsilon_{l}\right)=E^{\prime} \ell\left(\epsilon, \epsilon^{\prime}\right)+J_{\ell} / \sqrt{m}+O_{P}(1 / m),
$$

where we use the notation $E^{\prime}$ to denote

$$
\iint \ell\left(\epsilon, \epsilon^{\prime}\right) f_{\epsilon}(\epsilon) f_{\epsilon}\left(\epsilon^{\prime}\right) d \epsilon d \epsilon^{\prime}
$$

noting that $X_{1}^{\dagger}, \cdots, X_{n}^{\dagger}$ are fixed. So using this for both $N_{1}^{\dagger}$ and $\Delta_{1}^{\dagger}$ we have

$$
\frac{N_{1}^{\dagger}}{\Delta_{1}^{\dagger}}=\frac{E^{\prime} N_{1}^{\dagger}}{E^{\prime} \Delta_{1}^{\dagger}}+J_{h} / \sqrt{m}+O_{P}(1 / m) \text {. }
$$

First consider $E^{\prime} \Delta_{1}^{\dagger}$. Recalling that $\epsilon_{2}, \cdots, \epsilon_{n}$ are iidrv with density $f_{\epsilon}$,

$$
E^{\prime} \Delta_{1}^{\dagger}=\frac{1}{\sqrt{2 \pi \tau^{2}}} \iint e^{-\left(X_{3}^{\dagger}-\rho_{0}^{2} X_{1}^{\dagger}-\rho_{0} \epsilon-\epsilon^{\prime}\right)^{2} / 2 \tau^{2}\left(1+\rho_{0}^{2}\right)} f_{\epsilon}(\epsilon) f_{\epsilon}\left(\epsilon^{\prime}\right) d \epsilon d \epsilon^{\prime} .
$$

Consider the change of variables $\zeta=\left(\epsilon-\rho_{0} \epsilon^{\prime}\right) / \sqrt{1+\rho_{0}^{2}}$ and $\xi=\left(\rho_{0} \epsilon+\right.$ $\left.\epsilon^{\prime}\right) / \sqrt{1+\rho^{2}}$, then

$$
E^{\prime} \Delta_{1}^{\dagger}=\frac{1}{\sqrt{2 \pi \tau^{2}}} \iint e^{-\left(\xi-\left(X_{3}^{\dagger}-\rho_{0}^{2} X_{1}^{\dagger}\right) / \sqrt{1+\rho_{0}^{2}}\right)^{2} / 2 \tau^{2}} f(\zeta, \xi) d \zeta d \xi,
$$

where $f(\zeta, \xi)=f_{\epsilon}\left(\left(\zeta+\rho_{0} \xi\right) / \sqrt{1+\rho_{0}^{2}}\right) f_{\epsilon}\left((-\rho \zeta+\xi) / \sqrt{1+\rho_{0}^{2}}\right)$. Now putting $v=\left(\xi-\left(X_{3}^{\dagger}-\rho_{0}^{2} X_{1}^{\dagger}\right) / \sqrt{1+\rho_{0}^{2}}\right) / \tau$,

$$
E^{\prime} \Delta_{1}^{\dagger}=\frac{1}{\sqrt{2 \pi}} \iint e^{-v^{2} / 2} f\left(\zeta, \tau v+\frac{X_{3}^{\dagger}-\rho_{0}^{2} X_{1}^{\dagger}}{\sqrt{1+\rho_{0}^{2}}}\right) d \zeta d v
$$

imsart-aos ver. 2011/12/06 file: ARsprevsup1.tex date: March 11, 2013 
For small $\tau$, assuming $f_{\epsilon}$ is bounded and has bounded derivatives of first and second order, a Taylor expansion gives

$$
\int e^{-v^{2} / 2} f\left(\zeta, \tau v+\frac{X_{3}^{\dagger}-\rho_{0}^{2} X_{1}^{\dagger}}{\sqrt{1+\rho_{0}^{2}}}\right) d v=f\left(\zeta, \frac{X_{3}^{\dagger}-\rho_{0}^{2} X_{1}^{\dagger}}{\sqrt{1+\rho_{0}^{2}}}\right)+O\left(\tau^{2}\right) .
$$

So, taking $\tau=O\left(m^{-1 / 2}\right)$, here and in the sequel,

$$
E^{\prime} \Delta_{1}^{\dagger}=f_{\xi}\left(\frac{X_{3}^{\dagger}-\rho_{0}^{2} X_{1}^{\dagger}}{\sqrt{1+\rho_{0}^{2}}}\right)+O_{P}(1 / m)
$$

where $f_{\xi}$ denotes the marginal density of $\xi$.

Now consider $E^{\prime} N_{1}^{\dagger}$. Write $z^{\prime}=z-\rho_{0}\left(X_{1}^{\dagger}+X_{3}^{\dagger}\right) /\left(1+\rho_{0}^{2}\right)$,

$E^{\prime} N_{1}^{\dagger}=\frac{1}{2 \pi \tau^{2}} \iiint h(z) e^{-\frac{\left(z^{\prime} \sqrt{1+\rho_{0}^{2}}-\zeta\right)^{2}}{2 \tau^{2}}-\frac{\left(\left(X_{3}^{\dagger}-\rho_{0}^{2} x_{1}^{\dagger}\right) / \sqrt{1+\rho_{0}^{2}}-\xi\right)^{2}}{2 \tau^{2}}} f(\zeta, \xi) d z d \zeta d \xi$

After changing variables to $u=\left(\sqrt{1+\rho_{0}^{2}} z^{\prime}-\zeta\right) / \tau$ and $v=\left(\xi-\left(X_{3}^{\dagger}-\right.\right.$ $\left.\left.\rho_{0}^{2} X_{1}^{\dagger}\right) / \sqrt{1+\rho_{0}^{2}}\right) / \tau$ and noting that $h(z)=h\left(\tau u+\zeta+\rho_{0}\left(X_{1}^{\dagger}+X_{3}^{\dagger}\right) / \sqrt{1+\rho_{0}^{2}}\right)$, then, assuming bounded derivatives of $h$, we can proceed as for $E^{\prime} \Delta_{1}$ to show

$$
E^{\prime} N_{1}^{\dagger}=\int h\left(\zeta+\frac{\rho_{0}\left(X_{1}^{\dagger}+X_{3}^{\dagger}\right.}{\sqrt{1+\rho_{0}^{2}}}\right) f\left(\zeta, \frac{-\left(X_{3}^{\dagger}-\rho_{0}^{2} X_{1}^{\dagger}\right)}{\sqrt{1+\rho_{0}^{2}}}\right) d \zeta+O_{P}(1 / m) .
$$

So

$$
\text { (11) } \frac{E^{\prime} N_{1}^{\dagger}}{E^{\prime} \Delta_{1}^{\dagger}}=\int h\left(\zeta+\frac{\rho_{0}\left(X_{1}^{\dagger}+X_{3}^{\dagger}\right)}{\sqrt{1+\rho_{0}^{2}}}\right) f\left(\zeta \mid \xi=\frac{X_{3}^{\dagger}-\rho_{0}^{2} X_{1}^{\dagger}}{\sqrt{1+\rho_{0}^{2}}}\right) d \zeta+O_{P}\left(\frac{1}{m}\right) .
$$

The lemma follows on comparing this to (9) and using (10).

2.2. Proof of Lemma 2. We have for $a_{n}>0$,

$$
P\left(R^{\dagger} \geq u+a_{n}\right)-P_{1} \leq P\left(R^{*} \geq u\right) \leq P\left(R^{\dagger} \geq u-a_{n}\right)+P_{1},
$$

where $P_{1}=P\left(\left|R^{\dagger}-R^{*}\right|>a_{n}\right)$.

First consider $P_{1}$ for $\bar{A}^{\dagger 2}-4 u^{2} \bar{B}^{\dagger}>0$. Note that

$$
X_{1}^{\dagger}=\epsilon_{1}^{\dagger} / \sqrt{1-\rho_{0}^{2}}=\left(\epsilon_{1}^{*}+\tau Z_{1}\right) / \sqrt{1-\rho_{0}^{2}}=X_{1}^{*}+\tau Z_{1} / \sqrt{1-\rho_{0}^{2}}
$$


and for $i=2, \cdots, n$,

$$
\begin{aligned}
X_{i}^{\dagger} & =\rho_{0} X_{i-1}^{\dagger}+\epsilon_{i}^{\dagger} \\
& =X_{i}^{*}+\tau\left(\frac{\rho_{0}^{i-1}}{\sqrt{1-\rho_{0}^{2}}} Z_{1}+\rho_{0}^{i-2} Z_{2}+\cdots+Z_{i}\right) \\
& =X_{i}^{*}+\tau Z_{i}^{*},
\end{aligned}
$$

where $Z_{1}, \cdots, Z_{n}$ are independent standard normal variables and $Z_{1}^{*}, \cdots, Z_{n}^{*}$ are zero mean normal variables with $\operatorname{cov}\left(Z_{i}^{*}, Z_{j}^{*}\right)=\rho_{0}^{i-j} /\left(1-\rho_{0}^{2}\right)$ for $i \geq j$, which are independent of all other variables. So

$$
\begin{aligned}
R^{\dagger} & =\frac{\sum_{i=2}^{n} X_{i}^{\dagger} X_{i-1}^{\dagger}}{X_{1}^{\dagger 2} / 2+\sum_{i=2}^{n-1} X_{i}^{\dagger 2}+X_{n}^{\dagger 2} / 2} \\
& =\left(R^{*}+\tau r_{1}\right)\left(1+2 \tau r_{2}\right)^{-1},
\end{aligned}
$$

where

$$
r_{1}=\frac{\sum_{i=2}^{n}\left(X_{i}^{*} Z_{i-1}^{*}+X_{i-1}^{*} Z_{i}^{*}\right)}{X_{1}^{* 2} / 2+\sum_{i=2}^{n-1} X_{i}^{* 2}+X_{n}^{* 2} / 2}+\tau \frac{\sum_{i=2}^{n} Z_{i}^{*} Z_{i-1}^{*}}{X_{1}^{* 2} / 2+\sum_{i=2}^{n-1} X_{i}^{* 2}+X_{n}^{* 2} / 2}
$$

and

$$
r_{2}=\frac{X_{1}^{*} Z_{1}^{*}+2 \sum_{i=2}^{n-1} X_{i}^{*} Z_{i}^{*}+X_{n}^{*} Z_{n}^{*}}{X_{1}^{* 2} / 2+\sum_{i=2}^{n-1} X_{i}^{* 2}+X_{n}^{* 2} / 2}+\tau \frac{Z_{1}^{* 2} / 2+\sum_{i=2}^{n-1} Z_{i}^{* 2}+Z_{n}^{* 2} / 2}{X_{1}^{* 2} / 2+\sum_{i=2}^{n-1} X_{i}^{* 2}+X_{n}^{* 2} / 2} .
$$

Conditional on $X_{1}^{*}, \cdots, X_{n}^{*}$, since $Z_{1}^{*}, \cdots, Z_{n}^{*}$ are normal with zero means and finite variances, the first terms in $r_{1}$ and $r_{2}$ are normal with variances of order $1 / n$ and the second terms in each of these are multiplied by $\tau=$ $O(1 / \sqrt{m})$, so there is a $\delta>0$ such that

$$
P\left(\left|r_{k}\right|>a_{n} / \tau\right)=P\left(\sqrt{n}\left|r_{k}\right|>n a_{n}\right)<e^{-\delta n^{2} a_{n}^{2}}, \quad k=1,2 .
$$

Further, since $\bar{A}^{\dagger 2}-4 u^{2} \bar{B}^{\dagger}>0, K^{\dagger}\left(t^{\dagger}(u), u\right)$ is bounded, so there is a constant $c$ such that

$$
P\left(R^{*}>u\right)>e^{-c n\left(u-\rho_{0}\right)^{2}} .
$$

Take $u-\rho_{0}=o\left(n^{-1 / 4}\right)$ and $a_{n}=n^{-3 / 4}$, then for $\delta^{\prime}<\delta$,

$$
P_{1} / P^{*}\left(R^{*}>u\right)<e^{-\delta^{\prime} \sqrt{n}} \text {. }
$$

Also

$$
\begin{aligned}
P^{\dagger}\left(R^{\dagger}>u+a_{n}\right) / P^{\dagger}\left(R^{\dagger}>u\right) & =\frac{\bar{\Phi}\left(\sqrt{m} W^{\dagger+}\left(u+a_{n}\right)\right)}{\bar{\Phi}\left(\sqrt{m} W^{\dagger+}(u)\right)}\left(1+O_{P}(1 / m)\right. \\
& =1+O_{P}\left(m\left(u-\rho_{0}\right) a_{n}+1 / m\right) \\
& ==1+O_{P}\left(m\left(u-\rho_{0}\right)^{4}+1 / m\right),
\end{aligned}
$$

which completes the proof of the lemma. 
2.3. Proof of Lemma 3. Consider

$$
\int h(z) g^{\#}\left(z \mid X_{2 i-1}, X_{2 i+}\right) d z=\frac{N_{i}^{\#}}{\Delta_{i}^{\#}},
$$

where, considering $i=1$ for notational convenience,

$$
\Delta_{1}^{\#}=\frac{1}{(n-1)^{2}} \sum_{k} \sum_{l} e^{-\left(X_{3}-\rho_{0}^{2} X_{1}-\rho_{0} \epsilon_{k}-\epsilon_{l}\right)^{2} / 2 \tau^{2}\left(1+\rho_{0}^{2}\right)}
$$

and

$N_{1}^{\#}=\sum_{k} \sum_{l} \int \frac{h(z)}{2 \pi \tau^{2}(n-1)^{2}} e^{-\frac{\left(1+\rho_{0}^{2}\right)\left(z^{\prime}-\left(\epsilon_{k}-\rho_{0} \epsilon_{l}\right) /\left(1+\rho_{0}^{2}\right)\right)^{2}}{2 \tau^{2}}-\frac{\left(X_{3}-\rho_{0}^{2} X_{1}-\rho_{0} \epsilon_{k}-\epsilon_{l}\right)^{2}}{2 \tau^{2}\left(1+\rho_{0}^{2}\right)}} d z$.

Each of these expessions can be written in the form

$$
\frac{2}{(n-1)(n-2)} \sum_{k<l} \ell\left(\epsilon_{k}, \epsilon_{l}\right)=E^{\prime} \ell\left(\epsilon, \epsilon^{\prime}\right)+J_{1 \ell} / \sqrt{m}+O_{P}(1 / m),
$$

where

$$
E^{\prime} \Delta_{i}^{\#}=E^{\prime} \ell\left(\epsilon, \epsilon^{\prime}\right)=\iint \ell\left(\epsilon, \epsilon^{\prime}\right) f_{\epsilon}(\epsilon) f_{\epsilon}\left(\epsilon^{\prime}\right) d \epsilon d \epsilon^{\prime}
$$

and

$$
J_{1 \ell} / \sqrt{m}=\frac{2}{n-1} \sum_{k} \int \ell\left(\epsilon_{k}, \epsilon\right) f_{\epsilon}\left(\epsilon^{\prime}\right) d \epsilon^{\prime}-E^{\prime} \ell\left(\epsilon, \epsilon^{\prime}\right)
$$

and we have used the ideas of projection for U-statistics as in Serfling (1980). So that $J_{1 \ell}$ is a random variable with zero expectation and finite variance.

We get a similar expression for $N_{1}^{\#}$, so

$$
\frac{N_{1}^{\#}}{\Delta_{1}^{\#}}=\frac{E^{\prime} N_{1}^{\#}}{E^{\prime} \Delta_{1}^{\#}}+J_{1 h} / \sqrt{m}+O_{P}(1 / m)
$$

Then

$$
\frac{1}{m} \sum_{i} J_{i h} / \sqrt{m}=O_{P}(1 / m)
$$

Further, as in Section 2.1, we can show that

$$
\frac{E^{\prime} N_{1}^{\#}}{E^{\prime} \Delta_{1}^{\#}}=\int h\left(\zeta+\frac{\rho_{0}\left(X_{1}+X_{3}\right)}{\sqrt{1+\rho_{0}^{2}}}\right) f\left(\zeta \mid \xi=\frac{X_{3}-\rho_{0}^{2} X_{1}}{\sqrt{1+\rho_{0}^{2}}}\right) d \zeta+O_{P}\left(\frac{1}{m}\right) .
$$




\section{References.}

Field, C. and Robinson, J. (2013). Relative Errors for Bootstrap Approximations of the Serial Correlation Coefficient. Ann. Statist.

Hu, Z., Petrov, V. V. and Robinson, J. (2007). On large deviations of sums of independent random variables. Comm. Statist. Theory Methods 36 1981-1992. MR2396550

Jensen, J. L. (1995). Saddlepoint approximations. Oxford Statistical Science Series 16. The Clarendon Press Oxford University Press, New York. Oxford Science Publications. MR1354837

Robinson, J., Höglund, T., Holst, L. and Quine, M. P. (1990). On approximating probabilities for small and large deviations in $\mathbf{R}^{d}$. Ann. Probab. 18 727-753. MR1055431

Serfling, R. J. (1980). Approximation theorems of mathematical statistics. John Wiley \& Sons Inc., New York. Wiley Series in Probability and Mathematical Statistics. MR595165

Department of Mathematics and Statistics DALHOUSIE UNIVERSITY

Halifax, Nova Scotia

CANADA B3H 3J5

E-MAIL: *field@mathstat.dal.ca
SChoOl of Mathematics And Statistics UNIVERSITY OF SYDNEY, NSW 2006 Australia

E-MAIL: †john.robinson@sydney.edu.au 